\title{
Ultra-clean carbon nanotube electron guns for use in space
}

Stephanie Getty, Todd King, Mary Li, Larry Hess, Nick Costen, and Paul Mahaffy

Reliable mass spectrometers developed for spaceflight instruments could benefit future terrestrial applications.

Interest in carbon nanotubes (CNTs) for field-emission applications has been growing due to their low operating voltages and power consumption. For the same reasons, spaceflight instrument developers recognize the appeal of CNT sources for in-situ tools, such as an electron-impact ionization mass spectrometer (MS). ${ }^{1}$

A robust, high-performance MS could enable missions for which a long and reliable lifetime is important, such as a longduration Mars rover, entry probes to the giant gas planets, or to study the upper atmosphere of Venus, Mars, Titan, or a cometary coma. Previously, we have shown that CNT cathodes exhibit favorable power consumption, but their lifetime is diminished by the use of polymeric assembly materials. ${ }^{2,3}$ Here, we report on efforts to improve the reliability of a modular CNT electron gun for use in mass spectrometry.

In an electron-impact MS, an electron beam ionizes a sample gas through momentum transfer and the ions can then be analyzed using electromagnetic fields. Instrument sensitivity is in part determined by the number of electrons and how efficiently they ionize the sample. As a result, a high-performance electron gun must generate at least microamps of current at the low energies (70-100eV) that are most efficient for ionization.

For use in spaceflight, the electron gun must possess maximum reliability but minimum contamination, and only the cleanest construction materials should be used. Previously, we found that CNT emission can persist for hundreds of hours, but the polymers used were likely to be detrimental to the instrument's lifetime.

Our new ultra-clean approach will provide the MS with a low background signal and low pressure in the cathode vicinity for a longer lifetime. Using patterning, etching, bonding, and alignment micro-fabrication protocols, we have constructed a highly redundant array of CNT towers that are


Figure 1. (a) Patterned micron-scale CNT emitter array bonded to extraction grid. (b) Microamps of current are produced at low extraction voltage. Fowler-Nordheim tunneling describes the extraction of electrons from the carbon nanotube using an electric field. $J=$ current density. $E=$ Electric field. $K$ values are constants. 




Figure 2. CNT cathode integrated with silicon lens elements. Inset image shows that the electron gun interfaces to the MS via metal shielding at the output.

precisely aligned with a closely spaced extraction grid to achieve emission at the low voltages required, as shown in Figure 1.

Other groups have fabricated CNT emitters and the grid using a single substrate ${ }^{4,5}$ or micro electromechanical systems (MEMS) pop-up techniques. ${ }^{6}$ We integrate a prefabricated CNT array and grid using a low-temperature process. One advantage is that the CNTs are patterned and grown under ideal conditions before mating to the grid, and the high-quality CNTs remain as-grown and unaffected by grid integration. Moreover, our procedure is compatible with other emitters.

We have recently packaged the cathode-grid component with electrostatic lenses into a complete electron gun, as pictured in Figure 2. Testing has revealed that this design produces microamps of current between 150 and 200V, a four-fold improvement over previous cathodes. To achieve our target voltage of $100 \mathrm{~V}$ we will reduce cathode-grid spacing, but our approach makes this straightforward.

Our modular, ultra-clean CNT electron gun design is capable of producing microamps of current at the low voltages demanded by electron-impact ionization MS. After further testing and development, we expect improvements to emitter lifetime, ionization efficiency, and reduced background signal, as compared to previous prototypes. We intend to use our findings to guide future modifications to the MS design for further enhanced sensitivity, reliability, and performance in terrestrial and space-based instruments.
The authors acknowledge support from the Goddard Space Flight Center (GSFC) IRAD and the NASA ASTID Programs.

\section{Author Information}

\author{
Stephanie Getty and Todd King \\ Materials Engineering Branch \\ NASA Goddard Space Flight Center (GSFC) \\ Greenbelt, MD
}

Stephanie Getty received her $\mathrm{PhD}$ from the University of Florida in 2001. She received the GSFC AETD Science and Technology Advancement Award in 2007 and the Kerley Award for innovative partnerships in 2008. Her current interests include using nanomaterials in advanced instruments for spaceflight.

Todd King received his PhD from the University of Wisconsin in 1996. His current interests include developing miniature timeof-flight MS for planetary science.

\section{Mary Li, Larry Hess, and Nick Costen \\ Detector Systems Branch \\ NASA GSFC \\ Greenbelt, MD}

Mary Li has been leading the development of MEMS devices at GSFC since 1999. She graduated from the University of Maryland in 1992 with a PhD in materials science and engineering.

Larry Hess received his PhD in physical chemistry from the State University of New York at Buffalo. He led substrate fabrication efforts for wafer-level integration of the James Webb Space Telescope microshutter array and is currently involved in a similar effort for the LANDSAT infrared detector array.

Nicholas Costen has been involved in packaging MEMS devices since 1999. He graduated from Virginia Polytechnic Institute and State University in 1989 with a degree in mechanical engineering.

\section{Paul Mahaffy}

Atmospheric Experiments Laboratory

NASA GSFC

Greenbelt, MD 
Paul Mahaffy is chief of the Atmospheric Experiments Laboratory at GSFC. He is principal investigator on MS development for the LADEE lunar orbiter, the MAVEN Mars mission to study atmospheric loss, and sample analysis on the Mars Science Laboratory to be launched in 2011.

\section{References}

1. T. T. King, S. A. Getty, P. A. Roman, F. A. Herrero, H. H. Jones, D. M. Kahle, B. Lynch, G. Suárez, W. B. Brinckerhoff, and P. R. Mahaffy, "Simulation of a miniature, low-power time-of-flight mass spectrometer for in situ analysis of planetary atmospheres", Proc. SPIE 6959, p. 69590E, 2008. doi:10.1117/12.780113

2. S. A. Getty, T. T. King, R. A. Bis, H. H. Jones, F. Herrero, B. A. Lynch, P. Roman, and P. R. Mahaffy, "Performance of a carbon nanotube field emission electron gun", Proc. SPIE 6556, p. 18, 2007. doi:10.1117/12.720995

3. S. A. Getty, R. A. Bis, S. Snyder, E. Gehrels, K. Ramirez, T. T. King, P. A. Roman, and P. R. Mahaffy, "Effect of nitrogen gas on the lifetime of carbon nanotube field emitters for electron-impact ionization mass spectrometry", Proc. SPIE 6959, p. 695907, 2008. doi: $10.1117 / 12.776914$

4. M. A. Guillorn, M. D. Hale, V. I. Merkulov, M. L. Simpson, G. Y. Eres, H. Cui, A. A. Puretzky, and D. B. Geohegan, "Integrally gated carbon nanotube field emission cathodes produced by standard microfabrication techniques", J. of Vac. Sci. and Technol. B 21, p. 957, 2003. doi:10.1116/1.1565343

5. H. M. Manohara, M. J. Bronikowski, M. Hoenk, B. D. Hunt, and P. H. Siegel, "High-current-density field emitters based on arrays of carbon nanotube bundles", J. of Vac. Sci. and Technol. B 23, p. 157, 2005. doi:10.1116/1.1850103

6. C. Bower, W. Zhu, D. Shalom, D. Lopez, L. H. Chen, P. L. Gammel, and S. Jin, "On-chip vacuum microtriode using carbon nanotube field emitters", App. Phys. Lett. 80, p. 3820, 2002. doi:10.1063/1.1480884 